\title{
Grupos de Lie e EquaÇÕes Diferenciais Ordinárias*
}

\author{
A.S. PINTO, P.N. SILVA ${ }^{\dagger}$ A.L.C. SANTOS ${ }^{\ddagger}$
}

\begin{abstract}
Resumo
O ensino de equações diferenciais ordinárias se restringe a apresentação de uma variedade de técnicas para solucionar tipos específicos de equações, tais como exatas, separáveis, homogêneas, etc. Isto faz com que estudantes imaginem que o estudo de equações diferenciais se resume a uma lista de técnicas para resolver tais equações. Veremos que há uma teoria que unifica e estende a maioria destes métodos estudados que aparentemente não estão relacionados.
\end{abstract}

\section{Introdução}

Cursos convencionais ${ }^{1}$ de equações diferenciais ordinárias abrangem uma variedade de técnicas para solucionar tipos específicos de equações, tais como exatas, separáveis, homogêneas, etc. Um estudante, após o curso, pode imaginar que o estudo de equações diferenciais se resume a uma lista de técnicas para resolver tais equações. Além disso, as técnicas vistas ao longo do curso, parecem não ter relação umas com as outras. Contudo, existe uma bela teoria que unifica e estende a maioria destes métodos estudados que aparentemente não estão relacionados.

Tal teoria foi apresentada pelo matemático norueguês Sophus Lie em seu trabalho Theorie der Transformationsgruppen [3] e explora as simetrias das equações diferenciais. Basicamente, o método consiste em encontrar um novo sistema de coordenadas no qual a equação diferencial é resolvida mais facilmente. Lie desenvolveu para equações diferenciais o que Abel e Galois fizeram para equações algébricas - uma metodologia para classificá-las e resolvê-las utilizando teoria de grupos.

\section{Simetrias de equações algébricas}

A fim de entender as simetrias de equações diferenciais, é útil considerar simetrias de objetos mais simples. De modo genérico, uma simetria de um objeto geométrico é uma transformação cuja ação mantém o objeto aparentemente inalterado. Um exemplo que ilustra bem uma simetria é o resultado da rotação de um triângulo equilátero no sentido anti-horário em torno do seu baricentro. A Figura 1 ilustra uma ação desta simetria. Após uma rotação de $2 \pi / 3$, o triângulo parece tal como era antes da rotação, portanto esta transformação é uma simetria. Rotações de $4 \pi / 3$ e $2 \pi$ também são simetrias do triângulo equilátero. De fato, rotacionar a $2 \pi$ equivale a fazer nada, pois cada ponto do objeto é mapeado em si próprio. A

\footnotetext{
* Palavras chave: Grupos de Lie, Equações Diferenciais Ordinárias, Simetrias.

†UERJ, alisson.pinto@gmail.com,nunes@ime.uerj.br

‡CEFET-RJ, andreluiz.cordeiro@gmail.com

${ }^{1} \mathrm{O}$ conteúdo desta seção foi produzido pela tradução livre e adaptação das referências Hydon [2], Starrett [4] e Yap [5]
} 
transformação que leva cada ponto em si mesmo é uma simetria de qualquer objeto geométrico: tal transformação é denominada simetria trivial.

Existem certas restrições para simetrias de objetos geométricos: cada simetria possui uma única inversa, que é por si só uma simetria. A ação combinada da simetria e sua inversa sobre o objeto o mantém inalterado. Por exemplo, seja $\Gamma$ uma rotação do triângulo equilátero em $2 \pi / 3$. Então $\Gamma^{-1}$ (a inversa de $\Gamma$ ) é uma rotação em $4 \pi / 3$. Por simplicidade, neste trabalho, assumiremos que $\Gamma$ é um difeomorfismo $\left(C^{\infty}\right)$ - uma aplicação diferenciável suave cuja inversa também é suave.

Uma simetria possui as seguintes propriedades:

(S1) Preserva a estrutura do objeto.

(S2) É um difeomorfismo.

(S3) É uma transformação que mapeia o objeto em si mesmo (Condição de simetria).

Figura 1: Triângulo equilátero sob ação de simetria de rotação.

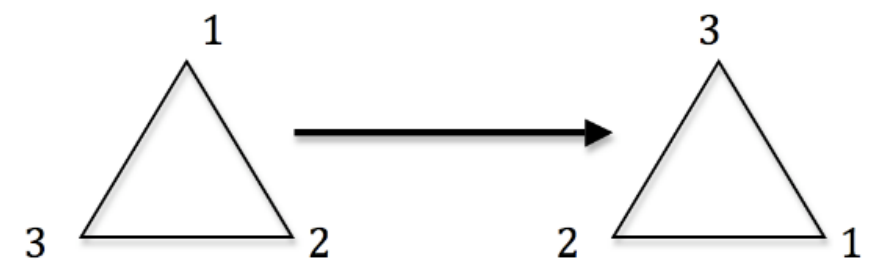

Fonte: [6], 2013.

No caso das equações diferenciais, queremos que as simetrias preservem suas soluções. Ao considerarmos o gráfico de $f(x)=x^{2}$ vemos que este é simétrico com respeito à reflexão em torno do eixo $y$, o gráfico de $g(x)=x^{3}$ é simétrico em relação à origem e o de $h(x)=\operatorname{sen} x$ é simétrico com respeito a uma translação horizontal de $2 \pi$. Nestes casos, as transformações do plano (reflexões e translações) são exemplos de simetrias que levam o gráfico das funções em si mesmo. Em geral, dada uma função $f: \mathbb{R} \rightarrow \mathbb{R}$, uma simetria de $f$ é uma aplicação contínua do $\mathbb{R}^{2}$ em $\mathbb{R}^{2}$ que leva o gráfico de $f$ em si mesmo e possui uma inversa contínua. Se $f$ é uma função de $\mathbb{R}^{2}$ em $\mathbb{R}$, uma simetria de $f$ é uma transformação definida em $\mathbb{R}^{3}$ que associa a qualquer ponto $(x, y, z)$ que satisfaz $z=f(x, y)$ um outro ponto de $\mathbb{R}^{3}$ que satisfaz a mesma equação, e assim por diante.

Considere agora a transformação $\Gamma_{\varepsilon}: \mathbb{R}^{2} \rightarrow \mathbb{R}^{2}$, dada por $\Gamma_{\varepsilon}(x, y)=\left(\varepsilon x, \varepsilon^{2} y\right), \forall \varepsilon \in \mathbb{R}$. $\Gamma_{\varepsilon}$ é uma simetria de $y=x^{2}$, pois se $(a, b)$ é um ponto no gráfico, temos que $\varepsilon^{2} b=\varepsilon^{2} a^{2}=(\varepsilon a)^{2}$, mostrando que $\Gamma_{\varepsilon}(a, b)=\left(\varepsilon a, \varepsilon^{2} b\right)$ também pertence ao gráfico. A Figura 2 mostra o comportamento desta simetria para valores específicos de $\varepsilon$. Se pensarmos em $\varepsilon$ como tempo, as letras e as curvas na Figura 2 permitem-nos acompanhar o movimento de pontos do plano sob a ação destas simetrias em vários instantes de tempo. 
Figura 2: Ação da simetria $\Gamma_{\varepsilon}(x, y)=\left(\varepsilon x, \varepsilon^{2} y\right)$

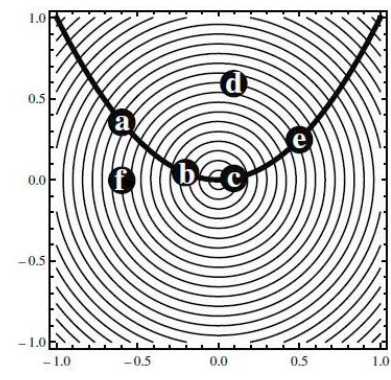

(a) $\varepsilon=0$

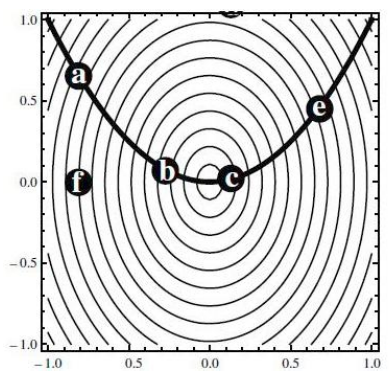

(b) $\varepsilon=0,3$

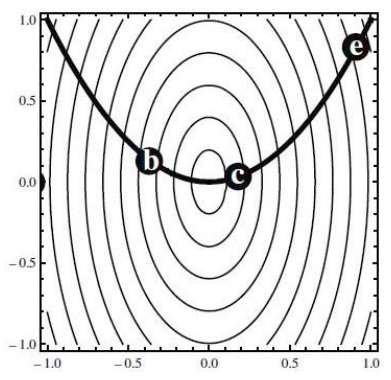

(c) $\varepsilon=0,6$

Fonte: [5], 2010.

Existem objetos que admitem uma quantidade finita de simetrias, como o triângulo equilátero. Outros possuem um conjunto infinito de simetrias. Por exemplo, para cada $\varepsilon \in(-\pi, \pi], \Gamma_{\varepsilon}:(x, y) \mapsto(\widehat{x}, \widehat{y})=$ $(x \cos \varepsilon-y \operatorname{sen} \varepsilon, x \operatorname{sen} \varepsilon+y \cos \varepsilon)$ é uma simetria do círculo unitário $x^{2}+y^{2}=1$, como mostra a Figura 3. Escrevendo em termos de coordenadas polares, temos $\Gamma_{\varepsilon}:(\cos \theta, \operatorname{sen} \theta) \mapsto(\cos (\theta+\varepsilon), \operatorname{sen}(\theta+\varepsilon))$. A transformação é uma rotação por $\varepsilon$ em torno do centro do círculo. A transformação preserva a estrutura do círculo e é suave e invertível (a inversa da rotação por $\varepsilon$ é $-\varepsilon$ ). Para provar que a condição de simetria é satisfeita, observe que $\widehat{x}^{2}+\widehat{y}^{2}=x^{2}\left(\cos ^{2} \varepsilon+\operatorname{sen}^{2} \varepsilon\right)+y^{2}\left(\cos \varepsilon^{2}+\operatorname{sen}^{2} \varepsilon\right)=x^{2}+y^{2}$, e portanto $\widehat{x}^{2}+\widehat{y}^{2}=1$ quando $x^{2}+y^{2}=1$.

Figura 3: Rotação do círculo de raio $r=1$.

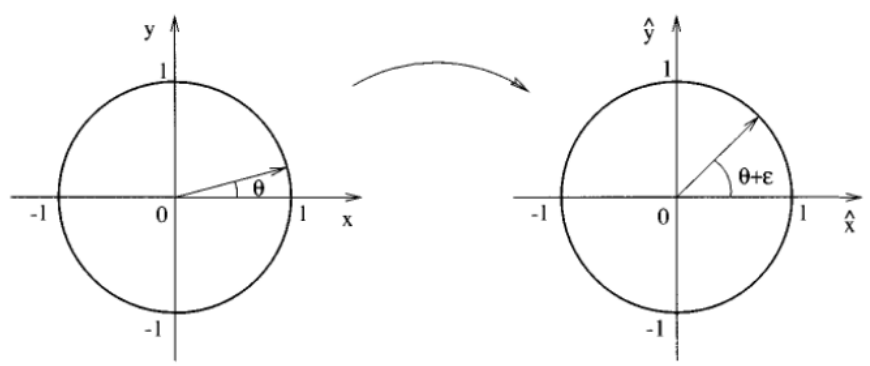

Fonte: [2], 2000.

$\mathrm{O}$ conjunto infinito de simetrias $\Gamma_{\varepsilon}$ é um exemplo de grupo de Lie a 1-parâmetro. Esta classe de simetrias a um parâmetro é imensamente útil e é a chave para construir soluções exatas de muitas equações diferenciais. 


\section{Simetrias de equações diferenciais}

Tal como em equações algébricas, as simetrias de equações diferenciais também permutam as soluções das equações.

Considere o conjunto de soluções da equação diferencial ordinária.

$$
\frac{d y}{d x}=0
$$

O conjunto de todas as soluções de (3.1) é formado pelas retas paralelas ao eixo $x$, ou seja $y(x)=$ $c, \forall c \in \mathbb{R}$, que cobrem o plano $x y$, tal como mostra a Figura 4. Qualquer simetria desta EDO deve, obrigatoriamente, levar uma solução particular em uma outra. Portanto, pela condição de simetria (S3), o conjunto das soluções no plano $x y$ deve ser indistinguível de sua imagem no plano $\hat{x} \hat{y}$. Logo, devemos ter

$$
\frac{d \hat{y}}{d \hat{x}}=0 \quad \text { quando } \quad \frac{d y}{d x}=0
$$

Figura 4: Soluções de $y^{\prime}=0$

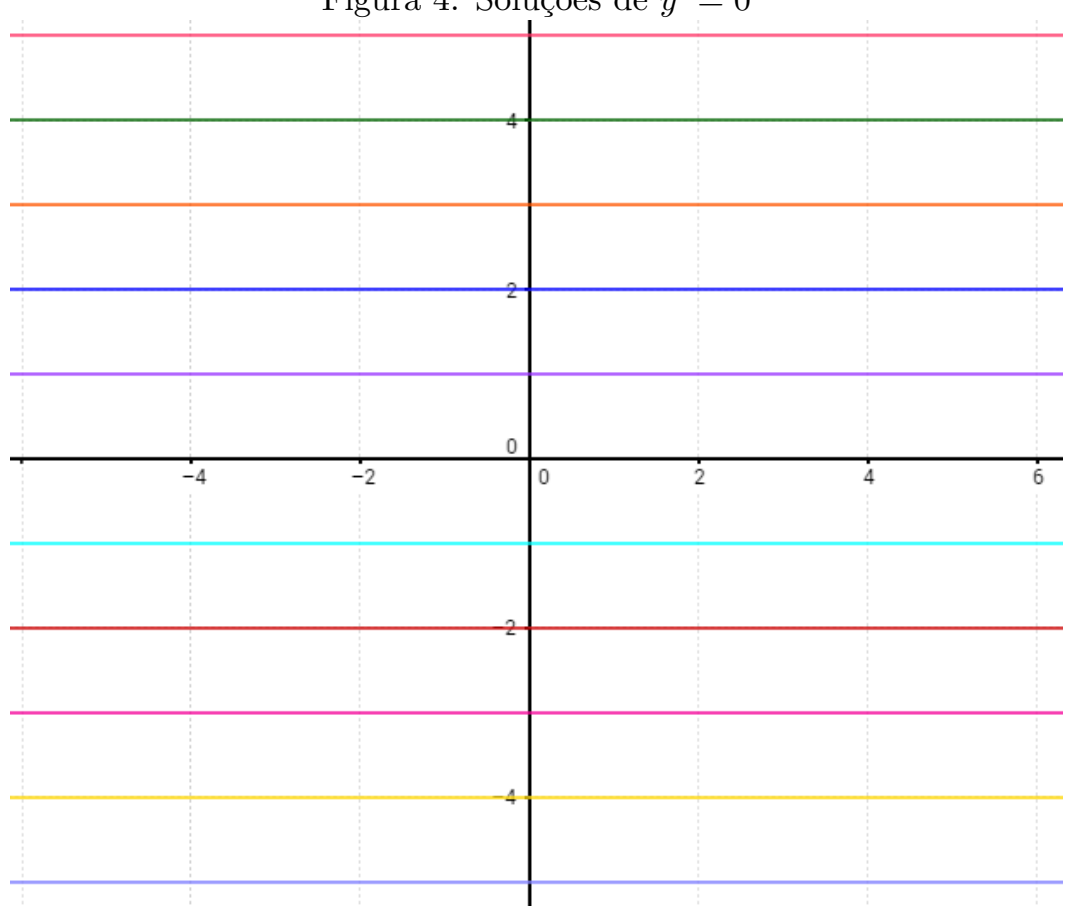

Fonte: O Autor, 2017.

Um exemplo de simetria para (3.1) é a simetria de escalas. Para um número real $\varepsilon$ qualquer, a aplicação

$$
\Gamma_{\varepsilon}:(x, y) \mapsto(\hat{x}, \hat{y})=\left(x, e^{\varepsilon} y\right)
$$

é uma simetria de (3.1) pois leva retas horizontais em outras retas semelhantes. Qualquer uma destas transformações, $\operatorname{com} \varepsilon \neq 0$, pode aumentar ou diminuir a distância entre as retas, mas não altera sua 
inclinação. No plano $\hat{x} \hat{y}$ as retas permanecem paralelas ao eixo $\hat{x}$, de modo que o conjunto de soluções é preservado.

Outros exemplos de simetrias para (3.1) são as translações, tanto na direção do eixo $x$ quanto do eixo $y$. A primeira pode ser definida por

$$
\Gamma_{\varepsilon}:(x, y) \mapsto(\hat{x}, \hat{y})=(x+\varepsilon, y)
$$

e a segunda

$$
\Gamma_{\varepsilon}:(x, y) \mapsto(\hat{x}, \hat{y})=(x, y+\varepsilon)
$$

Dada uma EDO de primeira ordem, nosso objetivo é encontrar um método geral para determinar um sistema de coordenadas, no qual a equação torna-se separável e possa ser resolvida por integração direta.

\subsection{Grupos de Lie}

Definition 3.1. Um conjunto $G$ de transformações $\Gamma_{\varepsilon}$ em $\mathbb{R}^{2}$ que dependem continuamente do parâmetro $\varepsilon$, onde $\varepsilon \in \mathbb{R}$, é um grupo a 1 -parâmetro se

(P1) $\Gamma_{\varepsilon}$ é uma bijeção.

(P2) $\Gamma_{\varepsilon} \circ \Gamma_{\delta}=\Gamma_{\varepsilon+\delta}, \quad \forall \varepsilon, \delta \in \mathbb{R}$.

(P3) apenas para $\varepsilon=0$, temos $\Gamma_{\varepsilon}=I$ (identidade).

(P4) Para cada $\varepsilon$, apenas para $\delta=-\varepsilon$, tem-se $\Gamma_{\varepsilon} \circ \Gamma_{\delta}=\Gamma_{0}=I$.

Vamos agora definir um grupo de simetrias para uma EDO de primeira ordem. Considere

$$
\frac{d y}{d x}=\omega(x, y)
$$

e um grupo $G$ de transformações a 1 -parâmetro $\Gamma_{\varepsilon}$

$$
(\widehat{x}, \widehat{y})=\Gamma_{\varepsilon}(x, y)=\mathcal{F}(x, y, \varepsilon)
$$

Definition 3.2. Um grupo $G$ de transformações a 1-parâmetro (3.6) é um grupo de simetrias da equação diferencial (3.5) se o conjunto de soluções de (3.5) é invariante sob a ação do grupo G. Isto é, cada elemento de $G$ permuta o conjunto de soluções de (3.5).

Se $G$ é um grupo de simetrias de $(3.5)$ e $\mathcal{F}$ em (3.6) é infinitamente diferenciável com respeito a $x$ e $y$ e analítica com respeito a $\varepsilon$, então $G$ é um grupo de Lie a 1 -parâmetro (ou transformação pontual de Lie). Neste caso, seus elementos são chamados de simetrias de Lie.

Exemplo 3.1. Como exemplo de um grupo de Lie a 1-parâmetro, temos a aplicação (3.2) que é uma simetria para cada $\varepsilon$ fixo para (3.1) e satisfaz todas as propriedades de grupos de simetria de Lie. 
De fato: Se dois pontos $\left(x_{1}, y_{1}\right)$ e $\left(x_{2}, y_{2}\right)$ são levados ao mesmo ponto $(\hat{x}, \hat{y})$, então $\left(x_{1}, y_{1}\right)=\left(x_{2}, y_{2}\right)$ e, para todo $(\hat{x}, \hat{y}) \in \mathbb{R}^{2}$, há um ponto $(x, y)$ aplicado em $(\hat{x}, \hat{y})$ por $\Gamma_{\varepsilon}$. Logo, a aplicação (3.2) é uma bijeção. O conjunto de transformações (3.2) satisfaz a propriedade de composição de grupos. Suponha $\Gamma_{\varepsilon_{2}} \circ \Gamma_{\varepsilon_{1}}$, ao aplicarmos $\Gamma_{\varepsilon_{1}}$ em $(x, y)$ temos $\left(\hat{x}_{1}, \hat{y}_{1}\right)=\left(x, e^{\varepsilon_{1}} y\right)$. Aplicando, em seguida, $\Gamma_{\varepsilon_{2}}$ em $\left(x, e^{\varepsilon_{1}} y\right)$ obtemos $\left(\hat{x}_{2}, \hat{y}_{2}\right)=\left(x, e^{\varepsilon_{1}+\varepsilon_{2}} y\right)$, que é o resultado da aplicação $\Gamma_{\varepsilon_{1}+\varepsilon_{2}}$. $\Gamma_{0}$ é a identidade pois, $(\hat{x}, \hat{y})=\left(x, e^{0} y\right)=(x, y)$. E finalmente, para todo $\varepsilon_{1} \in \mathbb{R}$ existe um único inverso. Efetuando a composição $\Gamma_{-\varepsilon_{1}} \circ \Gamma_{\varepsilon_{1}}$, temos $(\hat{x}, \hat{y})=\left(x, e^{-\varepsilon_{1}}\left(e^{\varepsilon_{1}} y\right)\right)=\left(x, e^{-\varepsilon_{1}+\varepsilon_{1}} y\right)=(x, y)$. Portanto, como todas as quatro propriedades da Definição 3.1 foram satisfeitas e já verificamos anteriormente invariância das soluções de (3.1), a aplicação (3.2) é um grupo de simetria para a equação (3.1). Mais ainda, como $\mathcal{F}(x, y, \varepsilon)=\left(x, e^{\varepsilon} y\right)$ é infinitamente diferenciável com respeito a $x$ e $y$ e analítica com respeito a $\varepsilon$, o conjunto de transformações (3.2) é um grupo de Lie a 1-parâmetro.

\subsection{Condição de simetria}

Seja $y(x)$ uma solução da equação diferencial ordinária de primeira ordem

$$
\frac{d y}{d x}=\omega(x, y)
$$

Uma simetria para esta equação leva a solução $y(x)$ a uma outra solução $\hat{y}(\hat{x})$. Isto significa que $\hat{y}(\hat{x})$ também é solução de

$$
\frac{d \hat{y}}{d \hat{x}}=\omega(\hat{x}, \hat{y})
$$

Uma ferramenta importante no estudo de simetrias é o operador derivada total definido como

$$
D_{x}=\frac{\partial}{\partial x}+\frac{d y}{d x} \frac{\partial}{\partial y}+\frac{d^{2} y}{d x^{2}} \frac{\partial}{\partial y^{\prime}} .
$$

Tal operador realiza a derivação implícita quando aplicado a uma equação em $x, y(x), y^{\prime}(x)$, etc.

Escrevendo (3.8) com o operador derivada total, temos

$$
\frac{d \hat{y}}{d \hat{x}}=\frac{D_{x} \hat{y}}{D_{x} \hat{x}}=\frac{\frac{\partial \hat{y}}{\partial x}+\frac{d y}{d x} \frac{\partial \hat{y}}{\partial y}}{\frac{\partial \hat{x}}{\partial x}+\frac{d y}{d x} \frac{\partial \hat{x}}{\partial y}}=\frac{\hat{y}_{x}+\frac{d y}{d x} \hat{y}_{y}}{\hat{x}_{x}+\frac{d y}{d x} \hat{x}_{y}}=\omega(\hat{x}, \hat{y})
$$

Um grupo de transformações é uma simetria quando a EDO, escrita nas coordenadas $\hat{x} \hat{y}$, não tem sua forma alterada. Ou seja

$$
\omega(\hat{x}, \hat{y})=\frac{\hat{y}_{x}+\omega(x, y) \hat{y}_{y}}{\hat{x}_{x}+\omega(x, y) \hat{x}_{y}} .
$$

Quando tal condição é satisfeita, dizemos que a equação diferencial é invariante. Considere o seguinte exemplo:

Exemplo 3.2. Vamos mostrar que a EDO de Riccati

$$
\frac{d y}{d x}=\frac{y+1}{x}+\frac{y^{2}}{x^{3}}
$$

é invariante sob o conjunto de transformações $\Gamma_{\varepsilon}(x, y) \mapsto(\hat{x}, \hat{y})=\left(\frac{x}{1-\varepsilon x}, \frac{y}{1-\varepsilon x}\right)$. 
Primeiramente calculamos as derivadas de $\hat{x}$ e $\hat{y}$ :

$$
\hat{x}_{x}=\frac{1}{(1-\varepsilon x)^{2}}, \quad \hat{x}_{y}=0, \quad \hat{y}_{x}=\frac{\varepsilon y}{(1-\varepsilon x)^{2}} \quad \text { e } \quad \hat{y}_{y}=\frac{1}{1-\varepsilon x} .
$$

Após o cálculo das derivadas, substituímos os resultados encontrados no lado direito de (3.10)

$$
\frac{\frac{\varepsilon y}{(1-\varepsilon x)^{2}}+\frac{1}{1-\varepsilon x}\left(\frac{y+1}{x}+\frac{y^{2}}{x^{3}}\right)}{\frac{1}{(1-\varepsilon x)^{2}}}=\frac{y+1-\varepsilon x}{x}+\frac{y^{2}}{x^{3}}(1-\varepsilon x) .
$$

No lado esquerdo, temos

$$
\omega(\hat{x}, \hat{y})=\frac{\hat{y}+1}{\hat{x}}+\frac{\hat{y}^{2}}{\hat{x}^{3}}=\frac{(y+1-\varepsilon x)}{x}+\frac{y^{2}}{x^{3}}(1-\varepsilon x) .
$$

Comprovando a igualdade de ambos os lados.

Em geral, é difícil calcular simetrias de uma EDO através da condição de simetria (3.10) devido a sua não-linearidade. Entretanto, é possível utilizar a expansão por série de Taylor para linearizar (3.10) e construir uma simetria.

Veremos agora alguns exemplos em que é possível usar a condição de simetria para encontrar uma simetria $^{2}$ para uma EDO dada.

Exemplo 3.3. A condição de simetria (3.10) para a EDO

$$
\frac{d y}{d x}=y
$$

é dada por

$$
\frac{\hat{y}_{x}+y \hat{y}_{y}}{\hat{x}_{x}+y \hat{x}_{y}}=\hat{y}
$$

Considerando $(\hat{x}, \hat{y})=(\hat{x}(x, y), y)$ e efetuando as derivadas que aparecem na equação $(3.10)$, temos

$$
\frac{y}{\hat{x}_{x}+y \hat{x}_{y}}=y \text {. }
$$

Simplificando a expressão acima, encontramos

$$
y=y\left(\hat{x}_{x}+y \hat{x}_{y}\right)
$$

De onde concluímos que para os dois lados serem iguais,

$$
\hat{x}_{x}+y \hat{x}_{y}=1
$$

Buscamos uma simetria que satisfaça a condição de simetria (3.10), tendo em mente (3.12). Considerando $\hat{x}_{y}=0$, a equação (3.12) torna-se $\hat{x}_{x}=1$, que é uma equação diferencial linear cuja solução é $\hat{x}=x+\varepsilon$, com $\varepsilon$ cumprindo papel de constante de integração. Portanto, todos os elementos do conjunto das translações

$$
\Gamma_{\varepsilon}:(x, y) \mapsto(\hat{x}, \hat{y})=(x+\varepsilon, y), \quad \varepsilon \in \mathbb{R}
$$

\footnotetext{
${ }^{2}$ Nos exemplos apresentados apresentamos famílias a 1-parâmetro de transformações $(\widehat{x}, \widehat{y})=\Gamma_{\varepsilon}(x, y)=\mathcal{F}(x, y, \varepsilon)$ e deixamos para o leitor a verificação das demais propriedades que garantem que elas se constituem em um grupo de Lie a 1-parâmetro.
} 
satisfazem (3.10).

Exemplo 3.4. Seja a equação diferencial

$$
\frac{d y}{d x}=\frac{1-y^{2}}{x}
$$

Queremos encontrar uma simetria de (3.13) que seja da forma

$$
(\hat{x}, \hat{y})=(\hat{x}(x), y)
$$

Como $\hat{y}=y, \hat{y}_{x}=\hat{x}_{y}=0$ e $\hat{y}_{y}=1$ em (3.10) encontramos que

$$
\frac{0+\frac{1-y^{2}}{x} \cdot 1}{\hat{x}_{x}+\frac{1-\hat{y}^{2}}{x} \cdot 0}=\frac{1-\hat{y}^{2}}{\hat{x}}=\frac{1-y^{2}}{\hat{x}}
$$

de modo que a condição de simetria para (3.13) se reduz a

$$
x \hat{x}_{x}=\hat{x}
$$

cuja solução, por integração direta, é $\hat{x}=e^{\varepsilon} x$, tendo $\varepsilon$ como constante de integração. Logo,

$$
\Gamma_{\varepsilon}:(x, y) \mapsto(\hat{x}, \hat{y})=\left(e^{\varepsilon} x, y\right)
$$

é uma simetria de (3.13).

As soluções não-constantes de (3.13) são dadas por

$$
y_{c}(x)=\frac{c x^{2}-1}{c x^{2}+1}, \quad c>0
$$

A Figura 5 mostra o comportamento de soluções de (3.13) sob ação da simetria (3.14) para valores específicos $\varepsilon$

Figura 5: O comportamento de diversas soluções sob ação da simetria $\Gamma_{\varepsilon}:(x, y) \mapsto\left(e^{\varepsilon} x, y\right)$ em vários pontos na órbita.

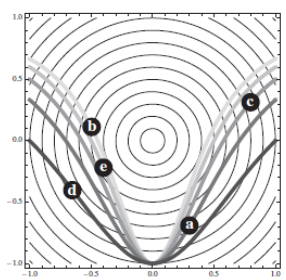

(a) $\varepsilon=1$.

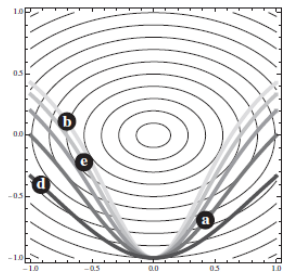

(b) $\varepsilon=1,5$.

Fonte: [5], 2010.

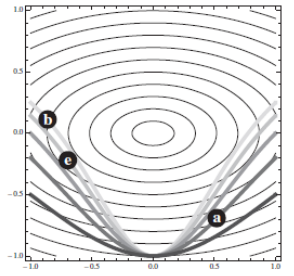

(c) $\varepsilon=2$. 


\section{Encontrando soluções de EDOs por meio de simetrias}

O modo como as simetrias são utilizadas para resolver equações diferenciais remetem a uma estratégia recorrente em matemática: problemas complicados podem se tornar mais simples ao serem visualizados em outros sistemas de coordenadas. Nesta seção é apresentado o método de solução de EDOs por meio de simetrias de translação e como uma simetria qualquer pode ser convertida numa simetria de translação.

Qualquer EDO que possua simetria da forma

$$
(\hat{x}, \hat{y})=(x, y+\varepsilon)
$$

pode ser resolvida utilizando técnicas de integração. Para todo $\varepsilon$ em alguma vizinhança de 0 , a condição de simetria (3.10) se reduz a

$$
\omega(x, y)=\omega(\hat{x}, \hat{y})=\omega(x, y+\varepsilon)
$$

Derivando (4.15) com respeito a $\varepsilon \operatorname{com} \varepsilon=0$, no lado esquerdo temos que

$$
\frac{d}{d \varepsilon} \omega(x, y)=0
$$

Para o lado direito, usando a regra da cadeia para derivar $\omega(x, y+\varepsilon)$ com respeito a $\varepsilon$, temos

$$
\frac{d}{d \varepsilon} \omega(x, y+\varepsilon)=\frac{\partial \omega}{\partial x} \cdot \frac{\partial x}{\partial \varepsilon}+\frac{\partial \omega}{\partial y} \cdot \frac{\partial y}{\partial \varepsilon}=\frac{\partial \omega}{\partial y} .
$$

Assim,

$$
\frac{\partial \omega}{\partial y}=0
$$

Logo, o lado direito da EDO original é uma função apenas de $x$

$$
\frac{d y}{d x}=\omega(x)
$$

Logo

$$
y=\int \omega(x) d x+c .
$$

Como nem todas as equações diferenciais possuem simetrias da forma (4.15), nosso interesse é encontrar outro sistema de coordenadas cuja simetria seja desta forma. Para tal, uma mudança de coordenadas deve ser feita. Considere o seguinte exemplo:

\section{Exemplo 4.1.}

$$
\frac{d y}{d x}=\frac{y^{3}+x^{2} y-x-y}{x^{3}+x y^{2}-x+y}
$$

À primeira vista, pode parecer bem difícil de resolver esta EDO. Contudo, uma mudança de variáveis para coordenadas polares $x=r \cos \theta$ e $y=r \operatorname{sen} \theta$, seguida das devidas simplificações, torna a tarefa mais simples. Efetuando a mudança de variáveis no lado direito da equação, temos

$$
\frac{(r \operatorname{sen} \theta)^{3}+(r \cos \theta)^{2} r \operatorname{sen} \theta-r \cos \theta-r \operatorname{sen} \theta}{(r \cos \theta)^{3}+r \cos \theta(r \operatorname{sen} \theta)^{2}-r \cos \theta+r \operatorname{sen} \theta}=\frac{r^{3} \operatorname{sen} \theta\left(\operatorname{sen}^{2} \theta+\cos ^{2} \theta\right)-r(\operatorname{sen} \theta+\cos \theta)}{r^{3} \cos \theta\left(\operatorname{sen}^{2} \theta+\cos ^{2} \theta\right)+r(\operatorname{sen} \theta-\cos \theta)}
$$


Considerando a identidade trigonométrica fundamental $\operatorname{sen}^{2} \theta+\cos ^{2} \theta=1$, pondo $r$ em evidência chegamos a

$$
\frac{\left(r^{2}-1\right) \operatorname{sen} \theta-\cos \theta}{\left(r^{2}-1\right) \cos \theta+\operatorname{sen} \theta},
$$

correspondendo ao lado direito da equação (4.16). No lado esquerdo, temos

$$
d x=\frac{\partial x}{\partial r} d r+\frac{\partial x}{\partial \theta} d \theta=\cos \theta d r-r \operatorname{sen} d \theta
$$

e

$$
d y=\frac{\partial y}{\partial r} d r+\frac{\partial y}{\partial \theta} d \theta=\operatorname{sen} \theta d r+r \cos d \theta
$$

Então, (4.16) se torna

$$
\frac{\operatorname{sen} \theta d r+r \cos d \theta}{\cos \theta d r-r \operatorname{sen} d \theta}=\frac{\operatorname{sen} \theta\left(r^{2}-1\right)-\cos \theta}{\cos \theta\left(r^{2}-1\right)+\operatorname{sen} \theta} .
$$

Queremos agora reescrevê-la em termos de $\frac{d r}{d \theta}$. Multiplicando cruzado a expressão acima, e simplificando a expressão chegamos a

$$
r\left(r^{2}-1\right) d \theta+d r=0,
$$

encontrando a equação diferencial separável nas coordenadas $r$ e $\theta$

$$
\frac{d r}{d \theta}=r\left(1-r^{2}\right)
$$

Resolvendo (4.17) por técnica de frações parciais temos que

$$
\theta=\ln \frac{r}{\sqrt{1-r^{2}}}+\varepsilon
$$

Como o lado direito de (4.17) independe de $\theta$, vemos que (4.17) admite a simetria

$$
(\hat{r}, \hat{\theta})=(r, \theta+\varepsilon)
$$

De fato, pela condição de simetria (3.10)

$$
\frac{d \hat{r}}{d \hat{\theta}}=\frac{\hat{r}_{\theta}+\frac{d r}{d \theta} \hat{r}_{r}}{\hat{\theta}_{\theta}+\frac{d r}{d \theta} \hat{\theta}_{r}}=\hat{r}\left(1-\hat{r}^{2}\right) .
$$

Logo, as soluções de (4.17) são invariantes sob o grupo contínuo de transformações $(r, \theta) \mapsto(r, \theta+\varepsilon)$ que representam, no plano $x y$, rotações em torno da origem conforme é mostrado na Figura 6 .

O Exemplo 4.1 mostra como resolver EDOs com simetrias de translação. Vamos mostrar futuramente como uma simetria geral pode ser levada à uma simetria de translação. Suponha que $y^{\prime}(x)=\omega(x, y)$ é simétrica com respeito à translações na direção de $y: \Gamma_{\varepsilon}(x, y) \mapsto(\hat{x}, \hat{y})=(x, y+\varepsilon)$. Então

$$
\omega(x, y+\varepsilon)=\omega(\hat{x}, \hat{y})=\frac{d \hat{y}}{d \hat{x}}=\frac{d(y+\varepsilon)}{d x}=\omega(x, y),
$$


mostrando que $\omega$ é independente de $y$. Portanto, $y^{\prime}(x)=\omega(x)$ é prontamente resolvida pela integração $y(x)=\int \omega(x) d x$, mostrando que qualquer equação diferencial com simetria de translação na direção de $y$ é separável.

Figura 6: Soluções da equação (4.16)

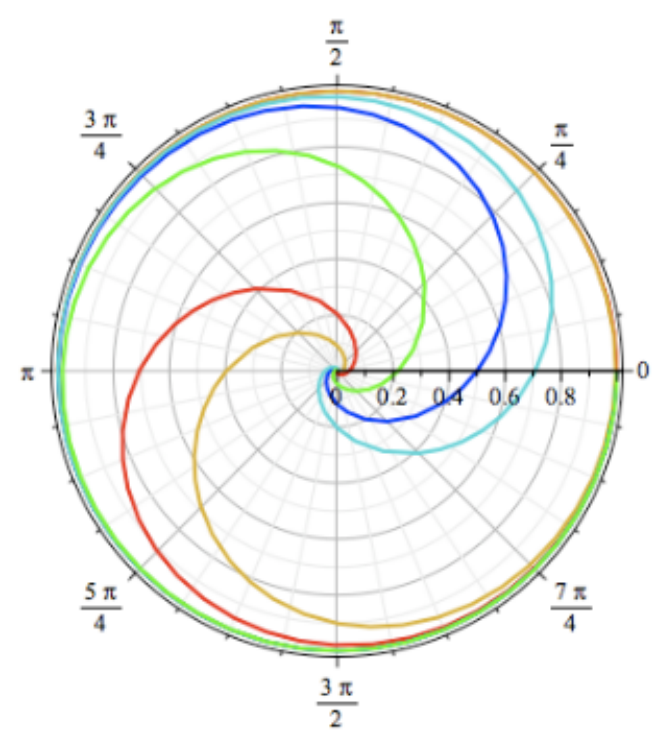

Fonte: [6], 2013.

\section{1 Órbitas}

Considere um ponto particular $\left(x_{0}, y_{0}\right)$ e a ação de um grupo de Lie $\Gamma_{\varepsilon}$. Quando $\varepsilon$ varia, o ponto $\left(\hat{x}_{0}, \hat{y}_{0}\right)=\Gamma_{\varepsilon}\left(x_{0}, y_{0}\right)$ move-se sobre o plano traçando uma curva contínua. Esta curva é chamada de órbita de $\left(x_{0}, y_{0}\right)$ sob o grupo, ou somente órbita do grupo. Se um grupo de Lie é uma simetria para a equação diferencial $\frac{d y}{d x}=\omega(x, y)$, então uma órbita do grupo forma um caminho contínuo transversal às curvas de soluções da equação diferencial.

Uma órbita de um ponto particular $\left(x_{0}, y_{0}\right)$ em uma solução é candidata à uma das coordenadas em um sistema de coordenadas no qual a equação diferencial torna-se fácil de ser integrada, pois nesta "direção" as curvas "deslizam" umas às outras.

Definition 4.1. O conjunto de pontos $\left\{\Gamma_{\varepsilon}(x, y) \mid a<\varepsilon<b\right\}$ traça uma curva no plano chamada órbita de $\Gamma_{\varepsilon}$. As coordenadas dos pontos sobre uma órbita através de $(x, y)$ são dadas por

$$
(\hat{x}, \hat{y})=(\hat{x}(x, y ; \varepsilon), \hat{y}(x, y ; \varepsilon)), \varepsilon \in(a, b)
$$

com

$$
(\hat{x}(x, y ; 0), \hat{y}(x, y ; 0))=(x, y)
$$


A órbita pode ser imaginada como um fluxo, pois cada ponto sobre o plano pode ser pensado como uma molécula de um fluido deslocando-se ao longo de uma trajetória definida por $\Gamma_{\varepsilon}$.

Uma simetria de (4.16) em coordenadas cartesianas é

$$
(\hat{x}, \hat{y})=(x \cos \varepsilon-y \operatorname{sen} \varepsilon, x \operatorname{sen} \varepsilon+y \cos \varepsilon) .
$$

As órbitas dos pontos sobre as soluções da equação (4.16) são círculos. Seja um ponto $\left(x_{0}, y_{0}\right) \neq(0,0)$, sua órbita é dada por

$$
\hat{x}^{2}+\hat{y}^{2}=r^{2}=x_{0}^{2}+y_{0}^{2} .
$$

De fato, segue de (4.18) que $\hat{x}^{2}+\hat{y}^{2}=x^{2}+y^{2}$. Como o ponto $\left(x_{0}, y_{0}\right) \neq(0,0)$ está fixado, vemos que o novo ponto $(\hat{x}, \hat{y})$ e o ponto original $(x, y)$ são pontos em um círculo de raio $r$. Isto pode ser visto também se reescrevemos (4.18) como uma matriz de rotação por $\varepsilon$ aplicada no ponto $(x, y)$ :

$$
(\hat{x}, \hat{y})=(x \cos \varepsilon-y \operatorname{sen} \varepsilon, x \operatorname{sen} \varepsilon+y \cos \varepsilon)=\left[\begin{array}{cc}
\cos \varepsilon & -\operatorname{sen} \varepsilon \\
\operatorname{sen} \varepsilon & \cos \varepsilon
\end{array}\right]\left[\begin{array}{l}
x \\
y
\end{array}\right]
$$

Vemos que ao varia $\varepsilon$ com $(x, y)=\left(x_{0}, y_{0}\right)$, obtemos um círculo.

Apresentaremos agora mais um exemplos de grupo de simetrias de Lie e discutiremos mais adiante a importância do uso de um sistema de coordenadas canônicas para resolver equações diferenciais ordinárias de primeira ordem. Uma EDO que possua uma simetria da forma

$$
(\hat{x}, \hat{y})=(x, y+\varepsilon)
$$

pode ser resolvida através de quadraturas. Contudo, uma simetria desta forma não necessariamente existe em coordenadas cartesianas para uma dada equação diferencial, daí a importância de um sistema de coordenadas canônicas.

Exemplo 4.2. Considere a equação de Bernoulli

$$
y^{\prime}=\frac{y(x-y)}{x^{2}},
$$

Os gráficos de suas soluções são mostrados na Figura 7. Utilizando um método que será explicado adiante, encontramos um sistema de coordenadas canônicas $(r, s)=\left(x, \frac{-x}{y}\right)$. Neste sistemas, as soluções da EDO transformam-se uma nas outras na direção de uma das coordenadas. Quando escrevemos (4.19) nas novas coordenadas, utilizando o operador derivada total em $r$ e $s, D_{x} s / D_{x} r$, obtemos

$$
\frac{d s}{d r}=\frac{1}{r}
$$

cujo lado direito não depende de $s$. Portanto, as curvas de solução podem ser transladadas umas às outras na direção $s$ e (4.19) torna-se separável nestas novas variáveis. Resolvendo (4.20) encontramos $s=\ln r+C$ e substituindo nas coordenadas originais, a solução de $(4.19)$ é $y=x /(\ln x+C)$. 
Figura 7: Uma família de soluções da equação de Bernoulli, nas coordenadas canônicas $r=x$ e $s=\frac{-x}{y}$.

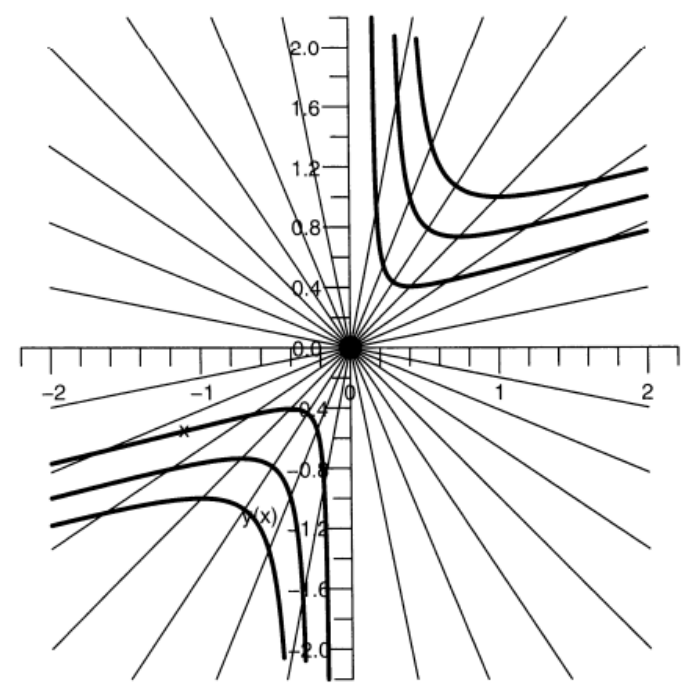

Fonte: [4], 2007.

\subsection{Vetor Tangente}

Suponha que $y^{\prime}(x)=\omega(x, y)$ é invariante sob algum grupo de Lie a 1-parâmetro que não seja necessariamente uma translação. Existe uma mudança de variáveis $(x, y) \mapsto(r, s)$, definida em algum domínio do $\mathbb{R}^{2}$, para o qual $y^{\prime}(x)=\omega(x, y)$ é invariante sob translações na direção $s$.

Definition 4.2. As tangentes à órbita em um ponto $(\hat{x}, \hat{y})$ qualquer são descritas por um vetor tangente, cuja abcissa denotada por

$$
\xi(\hat{x}, \hat{y})=\frac{d \hat{x}}{d \varepsilon}
$$

e cuja ordenada dada por

$$
\frac{d \hat{y}}{d \varepsilon}=\eta(\hat{x}, \hat{y})
$$

No ponto $(x, y)$, temos $\varepsilon=0$. Portanto,

$$
(\xi(x, y), \eta(x, y))=\left(\left.\frac{d \hat{x}}{d \varepsilon}\right|_{\varepsilon=0},\left.\frac{d \hat{y}}{d \varepsilon}\right|_{\varepsilon=0}\right) .
$$

As coordenadas do vetor tangente $\xi(x, y)$ e $\eta(x, y)$ podem ser usadas para encontrar um sistema de coordenadas simplificado. Contudo, $\xi(x, y)$ e $\eta(x, y)$ podem algumas vezes ser usados para encontrar uma curva de soluções sem o uso de diferentes coordenadas. Dizemos que uma solução da equação diferencial é invariante quando ela é sempre mapeada em si mesma sob a ação de uma simetria. Conforme $\varepsilon$ varia a solução é mapeada em si mesma e não em outra solução. Consequentemente, a órbita através de um ponto invariante coincide com a própria curva solução a que ele pertence. Neste caso, a derivada no 
ponto $(x, y)$ terá a mesma direção do vetor tangente e temos

$$
\frac{d y}{d x}=\omega(x, y)=\frac{\eta(x, y)}{\xi(x, y)}
$$

A equação característica $Q$ é definida por Hydon [2] como

$$
Q\left(x, y, y^{\prime}\right)=\eta(x, y)-y^{\prime} \xi(x, y)
$$

Como $\frac{d y}{d x}=\omega(x, y),(4.22)$ pode ser escrita como a equação característica reduzida

$$
\widetilde{Q}(x, y)=\eta(x, y)-\omega(x, y) \xi(x, y) .
$$

Se, sob uma dada simetria, a equação característica reduzida (4.23) for igual a 0, a curva solução da equação diferencial é invariante sob tal simetria.

Exemplo 4.3. A equação de Riccati

$$
\frac{d y}{d x}=x y^{2}-\frac{2 y}{x}-\frac{1}{x^{3}}, \quad x \neq 0
$$

possui a seguinte simetria

$$
(\hat{x}, \hat{y})=\left(e^{\varepsilon} x, e^{-2 \varepsilon} y\right)
$$

As componentes do vetor tangente são

$$
(\xi(x, y), \eta(x, y))=(x,-2 y)
$$

e a equação característica reduzida (4.23) se torna

$$
\widetilde{Q}(x, y)=-2 y-\left(x y^{2}-\frac{2 y}{x}-\frac{1}{x^{3}}\right) x=-x^{2} y^{2}+\frac{1}{x^{2}}
$$

Queremos determinar soluções da EDO (4.24) que sejam invariantes sob ação do grupo de simetrias (4.25). Segue de $\widetilde{Q}(x, y)=0$ que as soluções invariantes de $(4.24)$ são $y= \pm 1 / x^{2}$. Portanto, a simetria (4.25) age não-trivialmente para todas as soluções da equação (4.24), com exceção de $y=x^{-2}$ e $y=-x^{-2}$.

\subsection{Coordenadas Canônicas}

Geometricamente, converter uma simetria arbitrária em uma simetria de translação significa transformar as órbitas da simetria em órbitas da simetria de translação $(x, y) \mapsto(x, y+\varepsilon)$. Contudo, nem toda equação diferencial tem uma simetria desta forma em coordenadas cartesianas. Por isso, queremos mudar o sistema de coordenadas para que a simetria de translação seja possível. Como primeiro passo, temos que encontrar as coordenadas canônicas $(r(x, y), s(x, y))$ onde a equação diferencial torna-se separável. nas coordenadas canônicas, a equação diferencial será da forma $d s / d r=f(r)$ ou $d s / d r=f(s)$. Trataremos 
apenas o caso $d s / d r=f(r)$. Nas novas coordenadas há uma simetria $\Gamma_{\varepsilon}(r, s) \mapsto(\hat{r}, \hat{s})=(r, s+\varepsilon)$ que equivale a translações na direção $s[4,2007]$.

O vetor tangente no ponto $(r, s)$ será

$$
\left(\left.\frac{d \hat{r}}{d \varepsilon}\right|_{\varepsilon=0},\left.\frac{d \hat{s}}{d \varepsilon}\right|_{\varepsilon=0}\right)=(0,1) .
$$

Aplicando a regra da cadeia nas componentes do vetor tangente acima

$$
\left.\frac{d \hat{r}}{d \varepsilon}\right|_{\varepsilon=0}=\left.\frac{\partial \hat{r}}{\partial x} \frac{d x}{d \varepsilon}\right|_{\varepsilon=0}+\left.\frac{\partial \hat{r}}{\partial y} \frac{d y}{d \varepsilon}\right|_{\varepsilon=0}=\frac{\partial r}{\partial x} \xi(x, y)+\frac{\partial r}{\partial y} \eta(x, y)=0,
$$

e

$$
\left.\frac{d \hat{s}}{d \varepsilon}\right|_{\varepsilon=0}=\left.\frac{\partial \hat{s}}{\partial x} \frac{d x}{d \varepsilon}\right|_{\varepsilon=0}+\left.\frac{\partial \hat{s}}{\partial y} \frac{d y}{d \varepsilon}\right|_{\varepsilon=0}=\frac{\partial s}{\partial x} \xi(x, y)+\frac{\partial s}{\partial y} \eta(x, y)=1 .
$$

Isto é,

$$
r_{x} \xi(x, y)+r_{y} \eta(x, y)=0
$$

$$
s_{x} \xi(x, y)+s_{y} \eta(x, y)=1 .
$$

As equações (4.29) e (4.30) são EDPs lineares de primeira ordem para $r=r(x, y)$ e $s=s(x, y)$, podendo ser resolvidas pelo método das características [4]. Note que equação (4.29) satisfaz o produto interno

$$
\left\langle r_{x}, r_{y},-1\right\rangle \cdot\langle\xi, \eta, 0\rangle=0
$$

$\mathrm{O}$ vetor $\left\langle r_{x}, r_{y},-1\right\rangle$ é normal à superfície $z=r(x, y)$ no ponto $(x, y, r(x, y))$. Como o produto interno (4.31) é igual a zero, o vetor $(\xi(x, y), \eta(x, y), 0)$ é ortogonal ao vetor normal. Logo, $(\xi(x, y), \eta(x, y), 0)$ encontra-se no plano tangente ao gráfico de $r(x, y)$.

Seja $C$ uma curva parametrizada por $t \mapsto(x(t), y(t), z(t))$ sobre a superfície $z=r(x, y)$. Como $(\xi, \eta, 0)$ está no plano tangente, o vetor $(\xi(x(t), y(t)), \eta(x(t), y(t)), 0)$ é tangente à curva $C$. As equações características são

$$
\frac{d x}{d t}=\xi, \quad \frac{d y}{d t}=\eta \quad e \quad \frac{d z}{d t}=0
$$

de onde deduzimos

$$
\frac{d y}{d x}=\frac{\eta}{\xi}
$$

Repetindo o processo para (4.30), as equações características são:

$$
\frac{d x}{d t}=\xi, \quad \frac{d y}{d t}=\eta \quad e \quad \frac{d z}{d t}=1 .
$$

Lembrando que $z \equiv s$, temos:

$$
\frac{d x}{\xi(x, y)}=\frac{d y}{\eta(x, y)}=d s .
$$

Considere a função $r=r(x, y)=c$ e seja $y=y(x)$ uma solução de (4.32). Temos

$$
0=\frac{d r}{d x}=r_{x}+\frac{\eta}{\xi} r_{y}
$$


Logo, basta resolver (4.32) para determinar $r$.

Encontramos $s$ usando a equação (4.33)

$$
d s=\frac{d y}{\eta(x, y)}=\frac{d x}{\xi(x, y)}
$$

que resulta em

$$
s=\int \frac{d y}{\eta(x, y)}=\int \frac{d x}{\xi(x, y)} .
$$

No caso em que $\xi(x, y)=0$, tomamos $r=x$ e $s=\int \frac{d y}{\eta(x, y)}$.

As projeções das curvas de nível $r(x, y)=c, s(x, y)=k$ sobre o plano $x y$ formam a família de curvas que se tornarão o sistema de coordenadas simplificado, como mostra a Figura 8.

Figura 8: Superfície solução para uma EDP linear de primeira ordem com projeção de algumas de suas curvas de nível.

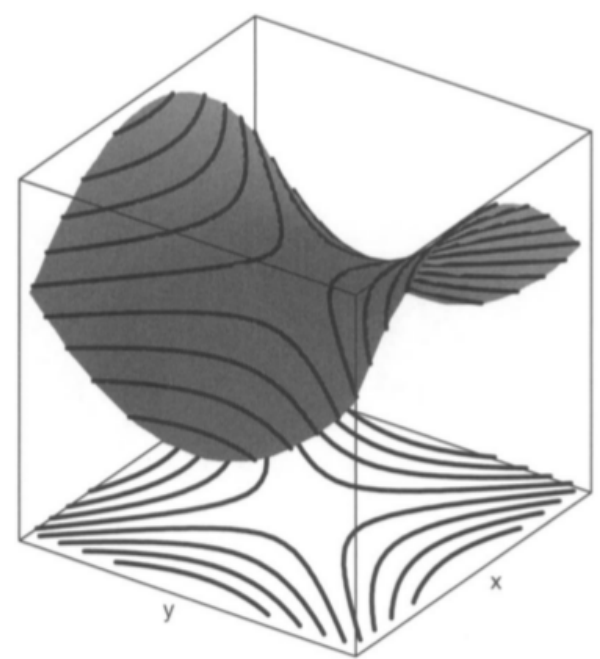

Fonte: [4], 2007.

Os vetores tangentes são utilizados para encontrar as coordenadas canônicas. Uma vez que os vetores tangentes e o sistema de coordenadas canônicas são determinados, simetrias podem ser reconstruídas a partir das coordenadas canônicas. Isto é feito, primeiramente, calculando as coordenadas canônicas $r(x, y)$ e $s(x, y)$ para $x$ e $y$ a fim de obter

$$
x=f(r, s) \quad \text { e } \quad y=g(r, s) .
$$

Logo, $\hat{x}$ e $\hat{y}$ são

$$
\hat{x}=f(\hat{r}, \hat{s})=f(r(x, y), s(x, y)+\varepsilon)
$$

e

$$
\hat{y}=g(\hat{r}, \hat{s})=g(r(x, y), s(x, y)+\varepsilon) .
$$


O objetivo é reescrever a equação diferencial em termos das novas coordenadas $r$ e $s$ para, em seguida, expressar sua solução nas coordenadas originais. Para encontrar $d s / d r$, aplicamos o operador derivada total (3.9) nas variáveis $r$ e $s$

$$
\frac{d s}{d r}=\frac{s_{x}+\omega(x, y) s_{y}}{r_{x}+\omega(x, y) r_{y}} .
$$

Esta operação resulta em uma equação $d s / d r$ escrita em termos de $x$ e $y$. Para escrevê-la em termos de $r$ e $s$ é necessário escrever $x$ e $y$ em termos de $r$ e $s$ e, em seguida, simplificar a equação. Após simplificar, resolvemos a equação em coordenadas canônicas e concluímos escrevendo a solução nas coordenadas originais $x y$.

Exemplo 4.4. A equação de Riccati

$$
\frac{d y}{d x}=\frac{y+1}{x}+\frac{y^{2}}{x^{3}}=\omega(x, y)
$$

admite o grupo de Lie de simetrias

$$
(\hat{x}, \hat{y})=\left(\frac{x}{1-\varepsilon x}, \frac{y}{1-\varepsilon y}\right) .
$$

Verificaremos apenas a condição de simetria (3.10). Primeiramente, calculamos as derivadas parciais

$$
\hat{y}_{x}=\frac{\varepsilon y}{\left(1-\varepsilon x^{2}\right)}, \quad \hat{y}_{y}=\frac{1}{1-\varepsilon x}, \quad \hat{x}_{x}=\frac{1}{(1-\varepsilon x)^{2}} \quad \text { e } \quad \hat{x}_{y}=0 .
$$

Substituindo as derivadas no lado direito da equação (3.10) temos

$$
\frac{\frac{\varepsilon y}{(1-\varepsilon x)^{2}}+\left(\frac{y+1}{x}+\frac{y^{2}}{x^{3}}\right) \frac{1}{1-\varepsilon x}}{\frac{1}{(1-\varepsilon x)^{2}}+\left(\frac{y+1}{x}+\frac{y^{2}}{x^{3}}\right) \cdot 0}=\frac{\hat{y}+1}{\hat{x}}+\frac{\hat{y}^{2}}{\hat{x}^{3}} .
$$

Logo, (3.10) é satisfeita.

As componentes do vetor tangente para esta simetria são

$$
(\xi(x, y), \eta(x, y))=\left(\left.\frac{d \hat{x}}{d \varepsilon}\right|_{\varepsilon=0},\left.\frac{d \hat{y}}{d \varepsilon}\right|_{\varepsilon=0}\right)=\left(x^{2}, x y\right) .
$$

Encontramos $r$ resolvendo a equação

$$
\frac{d y}{d x}=\frac{\eta(x, y)}{\xi(x, y)}=\frac{x y}{x^{2}}=\frac{y}{x}
$$

que é uma equação separável que, sendo integrada

$$
\int \frac{d y}{y}=\int \frac{d x}{x}
$$

resulta em

$$
\ln y=\ln x+c .
$$

Logo,

$$
y=c x
$$


Isolando a constante $c$ para encontrar $r$

$$
c=r=\frac{y}{x}
$$

encontramos a primeira coordenada canônica. Para encontrar $s$ basta calcular

$$
d s=\int \frac{d x}{\xi(x, y)}=\int \frac{d x}{x^{2}}=-\frac{1}{x} .
$$

Portanto, as coordenadas canônicas são

$$
(r, s)=\left(\frac{y}{x},-\frac{1}{x}\right) .
$$

O próximo passo é substituir as respectivas derivadas parciais

$$
s_{x}=\frac{1}{x^{2}}, \quad s_{y}=0, \quad r_{x}=-\frac{y}{x^{2}} \quad \text { e } \quad r_{y}=\frac{1}{x}
$$

no operador derivada total (4.34) nas coordenadas $r$ e $s$, resultando em

$$
\frac{d s}{d r}=\frac{\frac{1}{x^{2}}+\left(\frac{y+1}{x}+\frac{y^{2}}{x^{3}}\right) \cdot 0}{-\frac{y}{x^{2}}+\left(\frac{y+1}{x}+\frac{y^{2}}{x^{3}}\right) \cdot \frac{1}{x}} .
$$

Simplificando a equação acima, temos

$$
\frac{d s}{d r}=\frac{x^{2}}{x^{2}+y^{2}}=\frac{1}{1+\frac{y^{2}}{x^{2}}} .
$$

Reescrevendo o membro à direita em coordenadas canônicas obtemos

$$
\frac{d s}{d r}=\frac{1}{1+r^{2}}
$$

que é uma equação diferencial ordinária de primeira ordem separável. Resolvendo por integração direta

$$
s=\int \frac{d r}{1+r^{2}}
$$

obtemos

$$
s=\tan ^{-1}(r)+c .
$$

Agora que é conhecida a solução em coordenadas canônicas, a última etapa é escrever a solução nas coordenadas originais. Para tal, substituímos $(r, s)=(y / x,-1 / x)$

$$
\begin{aligned}
& \frac{-1}{x}=\tan ^{-1}\left(\frac{1}{x}\right)+c . \\
& \tan ^{-1}\left(\frac{y}{x}\right)=c-\frac{1}{x} \\
& \left(\frac{y}{x}\right)=\tan \left(-\frac{1}{x}+c\right)
\end{aligned}
$$

A solução da equação de Riccati é

$$
y(x)=x \tan \left(-\frac{1}{x}+c\right) .
$$


O comportamento das soluções da equação de Riccati pode ser visto na Figura 9.

Figura 9: $\mathrm{O}$ comportamento de diversas soluções sob ação da simetria $\Gamma_{\varepsilon}:(x, y) \mapsto\left(\frac{x}{1-\varepsilon x}, \frac{y}{1-\varepsilon x}\right)$ em vários pontos do plano.

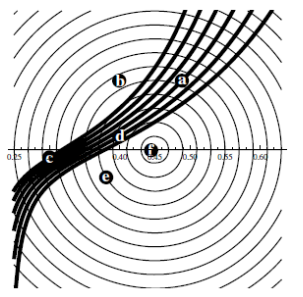

(a) $\varepsilon=0$

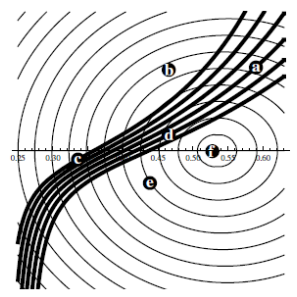

(b) $\varepsilon=0,3$

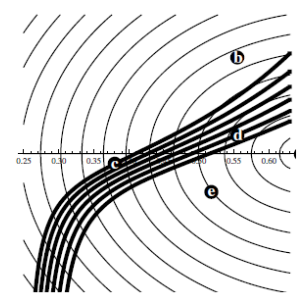

(c) $\varepsilon=0,6$

Fonte: [5], 2010.

O processo para resolver equações diferenciais, utilizando simetrias de Lie obedece ao seguinte algoritmo:

1. Calcular um conjunto de simetrias da equação diferencial;

2. Efetuar mudança de variáveis convertendo a simetria em uma simetria de translação;

3. Nas novas coordenadas, a EDO torna-se separável;

4. Integrar a EDO nas novas variáveis e expressar a solução nas variáveis originais.

\subsection{Condição de Simetria Linearizada}

Em todos os exemplos apresentados até aqui, a simetria necessária para resolver a equação diferencial foi dada. Todavia, é uma tarefa árdua encontrar uma simetria que funcione para uma equação diferencial dada. Para encontrar uma simetria, deve-se satisfazer a condição de simetria definida pela equação (3.10):

$$
\omega(\hat{x}, \hat{y})=\frac{\hat{y}_{x}+\omega(x, y) \hat{y}_{y}}{\hat{x}_{x}+\omega(x, y) \hat{x}_{y}}
$$

Esta equação resulta na simetria $(x, y) \mapsto(\hat{x}, \hat{y})$. Resolvendo $(3.10)$ para $\hat{x}$ e $\hat{y}$, é possível encontrar as componentes $\xi$ e $\eta$ do vetor tangente e, por meio delas, encontrar as novas coordenadas. Contudo, esta equação pode ser muito difícil de ser resolvida.

Para encontrar o vetor tangente $(\xi, \eta)$, expandimos $\hat{x}, \hat{y}$ e $\omega(x, y)$ em série de Taylor em torno do ponto $\varepsilon=0$, obtendo

$$
\begin{gathered}
\hat{x}=x+\xi(x, y) \varepsilon+\mathcal{O}\left(\varepsilon^{2}\right), \\
\hat{y}=y+\eta(x, y) \varepsilon+\mathcal{O}\left(\varepsilon^{2}\right)
\end{gathered}
$$

$\mathrm{e}$

$$
\omega(\hat{x}, \hat{y})=\omega(x, y)+\omega_{x}(x, y) \xi(x, y) \varepsilon+\omega_{y}(x, y) \eta(x, y) \varepsilon+\mathcal{O}\left(\varepsilon^{2}\right)
$$


Desprezando os termos de ordem 2 e superior, obtemos as linearizações de $\hat{x}, \hat{y}$ e $\omega(\hat{x}, \hat{y})$.

Conhecidas as linearizações, a próxima etapa é substituir suas respectivas derivadas na equação (3.10). De (4.35) temos

$$
\hat{x}_{x}=1+\varepsilon \xi_{x} \quad \text { e } \quad \hat{x}_{y}=\varepsilon \xi_{y} .
$$

De (4.36), temos

$$
\hat{y}_{x}=\varepsilon \eta_{x} \quad \text { e } \quad \hat{y}_{y}=1+\varepsilon \eta_{y} .
$$

A equação (3.10) torna-se

$$
\omega(\hat{x}, \hat{y})=\frac{d \hat{y}}{d \hat{x}}=\frac{\omega+\varepsilon\left(\eta_{x}+\omega \eta_{y}\right)}{1+\varepsilon\left(\xi_{x}+\omega \xi_{y}\right)} .
$$

Substituindo $\omega(\hat{x}, \hat{y})$ pela equação $(4.37)$

$$
\omega+\varepsilon\left(\omega_{x} \xi+\omega_{y} \eta\right)=\frac{\omega+\varepsilon\left(\eta_{x}+\omega \eta_{y}\right)}{1+\varepsilon\left(\xi_{x}+\omega \xi_{y}\right)} .
$$

Multiplicando ambos os membros da igualdade pelo denominador

$$
\omega+\varepsilon\left(\omega_{x} \xi+\omega_{y} \eta\right)\left(1+\varepsilon\left(\xi_{x}+\omega \xi_{y}\right)\right)=\omega+\varepsilon\left(\eta_{x}+\omega \eta_{y}\right) .
$$

Desconsiderando os termos de ordem 2 ou mais:

$$
\omega+\varepsilon\left(\omega_{x} \xi+\omega_{y} \eta\right)+\omega \varepsilon\left(\xi_{x}+\omega \xi_{y}\right)=\omega+\varepsilon\left(\eta_{x}+\omega \eta_{y}\right)
$$

Simplificando ainda mais,

$$
\eta_{x}+\omega \eta_{y}=\omega_{x} \xi+\omega_{y} \eta+\omega \xi_{x}+\omega^{2} \xi_{y},
$$

obtemos a condição de simetria linearizada

$$
\eta_{x}+\left(\eta_{y}-\xi_{x}\right) \omega-\xi_{y} \omega^{2}=\xi \omega_{x}+\eta \omega_{y}
$$

Exemplo 4.5. Seja a equação diferencial

$$
\frac{d y}{d x}=\frac{y}{x}+x
$$

Neste caso, $\omega(x, y)=\frac{y}{x}+x$. Substituindo $\omega$ e suas respectivas derivadas na condição de simetria linearizada, temos

$$
\eta_{x}+\left(\eta_{y}-\xi_{x}\right)\left(\frac{y}{x}+x\right)-\xi_{y}\left(\frac{y}{x}+x\right)^{2}-\left(\xi\left(1-\frac{y^{2}}{x^{2}}\right)+\eta\left(\frac{1}{x}\right)\right)=0 .
$$

Precisamos resolver esta equação para $\xi$ e $\eta$. Na forma como está, a equação ainda é bem complicada de ser resolvida. Em um primeiro momento, pode parecer ineficaz utilizar a condição de simetria linearizada para encontrar a simetria, já que este é o mesmo objetivo da equação (3.10). Para resolver a equação linearizada, fazemos uma suposição (damos um palpite!) sobre $\xi$ e $\eta$ : $\xi=0$ e $\eta$ é uma função apenas de $x(\eta(x))$. Temos, então, $\xi_{y}=0$ e $\xi_{x}=0=\xi_{y}$ e a equação linearizada para (4.39)

$$
\eta_{x}-\frac{\eta}{x}=0
$$


É simples encontrar a solução desta equação diferencial:

$$
\begin{gathered}
\int \frac{d \eta}{\eta}=\int \frac{d x}{x} \\
\ln \eta=\ln x+c_{0} \\
\eta=c x .
\end{gathered}
$$

Agora que o vetor tangente $(\xi, \eta)=(0, c x)$ é conhecido, podemos encontrar as coordenadas canônicas $r$ e $s$. Como $\xi(x, y)=0$, então $r=x$. Encontramos $s$ resolvendo

$$
\begin{gathered}
d s=\frac{d y}{\eta} \\
s=\int \frac{d y}{c x}=\frac{y}{c x} .
\end{gathered}
$$

Admitindo $c=1$, definimos as coordenadas canônicas

$$
(r(x, y), s(x, y))=\left(x, \frac{y}{x}\right)
$$

cujas respectivas derivadas parciais são

$$
r_{x}=1, \quad r_{y}=0, \quad s_{x}=-\frac{y}{x^{2}} \quad \text { e } \quad s_{y}=\frac{1}{x} .
$$

Substituindo $r$ e $s$ em (4.34) obtemos

$$
\frac{d s}{d r}=\frac{-\frac{y}{x^{2}}+\left(\frac{y}{x}+x\right)\left(\frac{1}{x}\right)}{1}=1 .
$$

Portanto, a equação nas coordenadas canônicas torna-se

$$
\frac{d s}{d r}=1
$$

cuja solução é $s=r+k, k$ constante. Escrevendo a solução nas coordenadas $x$ e $y$ :

$$
\frac{y}{x}=x+k .
$$

A solução geral da equação diferencial (4.39) é

$$
y=x^{2}+k x .
$$

\subsection{Geradores infinitesimais de simetrias}

Os métodos descritos nas seções anteriores podem ser utilizados para resolver equações diferenciais ordinárias de primeira ordem (3.7), possibilitando-nos discutir muitas das ideias geométricas que são a base dos métodos de simetria. Supondo que uma EDO de primeira ordem possua um grupo de Lie a 1 -parâmetro de simetrias, cujo vetor tangente em $(x, y)$ é $(\xi, \eta)$, o operador diferencial parcial

$$
\mathbf{X}=\xi \frac{\partial}{\partial x}+\eta \frac{\partial}{\partial y}
$$


é chamado gerador infinitesimal do grupo de Lie. As equações (4.27) e (4.28), que definem as coordenadas canônicas, podem ser reescritas como

$$
\mathbf{X} r=0, \mathbf{X} s=1 \text {. }
$$

O termo "gerador" indica que repetidas aplicações das transformações geram uma transformação finita, que é uma maneira diferente de expressar o fato de que as integrais de $\mathbf{X}$ são o grupo de órbitas. O gerador de simetrias pode ser visto como os vetores tangentes às órbitas das expansões (3.3) e (3.4), sendo $(\hat{x}, \hat{y})$ soluções do PVI

$$
\frac{d \widehat{x}}{d \epsilon}=\xi(d \widehat{x}, \widehat{y}), \quad \frac{d \widehat{y}}{d \epsilon}=\eta(d \widehat{x}, \widehat{y}), \quad(\widehat{x}, \widehat{y})=(x, y) \quad \text { quando } \epsilon=0
$$

A solução para este PVI pode ser escrita como as séries de potências

$$
\widehat{x}=e^{\epsilon X} x, \quad \widehat{y}=e^{\epsilon X} y,
$$

onde

$$
e^{\epsilon X}=\sum_{n=0}^{\infty} \frac{\epsilon^{n}}{n !} X^{n} .
$$

Logo, uma vez que $\mathbf{X}$ é conhecido, é possível calcular $(\hat{x}, \hat{y})$, tal que as órbitas possam ser encontradas.

A maioria dos métodos de simetria utilizam os vetores tangentes, em vez de simetrias. Entretanto, simetrias de Lie podem ser reconstruídas a partir dos vetores tangentes, integrando as EDOs sujeitas à condição inicial em (4.21). Então (localmente) há uma correspondência um a um entre cada grupo de Lie a 1 -parâmetro e seus vetores tangentes.

Seja um grupo de Lie de transformações $(\hat{x}, \hat{y})=(\hat{x}(x, y ; \varepsilon), \hat{y}(x, y ; \varepsilon))$. Expandindo $\hat{x}$ e $\hat{y}$ na série de Taylor em torno do ponto $\varepsilon=0$, encontramos

$$
\hat{x}=x+\left.\varepsilon \frac{d \hat{x}}{d \varepsilon}\right|_{\varepsilon=0}+O\left(\varepsilon^{2}\right)
$$

$$
\hat{y}=y+\left.\varepsilon \frac{d \hat{y}}{d \varepsilon}\right|_{\varepsilon=0}+O\left(\varepsilon^{2}\right)
$$

onde $\varepsilon=0$ constitui a transformação identidade do grupo. Definindo novas funções

$$
\xi(x, y)=\left.\frac{\partial \hat{x}}{\partial \varepsilon}\right|_{\varepsilon=0}, \quad \eta(x, y)=\left.\frac{\partial \hat{y}}{\partial \varepsilon}\right|_{\varepsilon=0}
$$

as expansões (4.42) e (4.43) tornam-se

$$
\hat{x}=x+\xi(x, y) \varepsilon, \quad \hat{y}=x+\eta(x, y) \varepsilon .
$$

Geometricamente, as transformações acima definem o vetor tangente $(\xi(x, y), \eta(x, y))$ no ponto $(x, y)$. Logo, $(\xi(x, y), \eta(x, y))$ é chamado de campo vetorial tangente do grupo.

A seguir são mostrados alguns exemplos de geradores infinitesimais para algumas simetrias vistas anteriormente. 
O grupo de rotações

$$
\Gamma_{\varepsilon}:(x, y) \mapsto(\hat{x}, \hat{y})=(x \cos \varepsilon-y \operatorname{sen} \varepsilon, x \operatorname{sen} \varepsilon+y \cos \varepsilon)
$$

possui infinitesimais definidos como

$$
\xi(x, y)=\left.\frac{\partial \hat{x}}{\partial \varepsilon}\right|_{\varepsilon=0}=-y, \quad \eta(x, y)=\left.\frac{\partial \hat{y}}{\partial \varepsilon}\right|_{\varepsilon=0}=x
$$

Portanto o gerador infinitesimal da simetria de rotação é

$$
\mathbf{X}=\xi(x, y) \frac{\partial}{\partial x}+\eta(x, y) \frac{\partial}{\partial y}=-y \frac{\partial}{\partial x}+x \frac{\partial}{\partial y} .
$$

O grupo de simetrias de translação nas direções $x$ e $y$ definidos como

$$
(\hat{x}, \hat{y})=(x+\varepsilon, y+\varepsilon)
$$

possui o gerador de simetrias

$$
\mathbf{X}=\frac{\partial}{\partial x}+\frac{\partial}{\partial y}
$$

pois $\xi=d \hat{x} / \hat{\varepsilon}=1$ e $\xi=d \hat{x} / \hat{\varepsilon}=1$ derivando em $\varepsilon=0$.

$\mathrm{O}$ conjunto de simetrias

$$
(\hat{x}, \hat{y})=\left(\frac{x}{1-\varepsilon x}, \frac{y}{1-\varepsilon y}\right)
$$

possui vetor tangente

$$
(\xi, \eta)=\left(\left.\frac{d \hat{x}}{d \varepsilon}\right|_{\varepsilon=0},\left.\frac{d \hat{y}}{d \varepsilon}\right|_{\varepsilon=0}\right)=\left(x^{2}, x y\right)
$$

Portanto, o gerador infinitesimal para este grupo é

$$
\mathbf{X}=x^{2} \frac{\partial}{\partial x}+x y \frac{\partial}{\partial y}
$$

\section{EDOs de primeira ordem invariantes em relação a um grupo de simetrias conhecido}

O conceito de invariante diferencial é bastante útil para a caracterização de famílias de EDOs que admitem certas simetrias. Para utilizá-lo, precisaremos de um conceito adicional: o de prolongamento de um grupo de simetrias $\Gamma:(x, y) \mapsto(\hat{x}, \hat{y})$.

Definition 5.1. O operador

$$
\mathbf{X}^{(m)}=\xi \partial_{x}+\eta \partial_{y}+\eta^{(1)} \partial_{y^{\prime}}+\cdots+\eta^{(m)} \partial_{y^{(m)}}
$$

onde $\eta^{(k)}$ é o termo de $O(\varepsilon)$ na expansão de $\hat{y}^{(k)}$ em torno de $\varepsilon=0$, é chamado de prolongamento de ordem $m$ do gerador infinitesimal $\mathbf{X}$. 
O prolongamento de ordem $m$ do gerador infinitesimal permite analisar a ação do grupo de simetrias sobre derivadas de ordem $m$ ou menor.

Definition 5.2. Uma função não constante $I\left(x, y, y^{\prime}, \ldots, y^{(k)}\right)$ é um invariante diferencial de ordem $k$ do grupo de simetrias gerado por $\mathbf{X}$ se

$$
\mathbf{X}^{(k)} I=0
$$

Dada uma simetria, os invariantes diferenciais podem ser usados para construir uma EDO que a admite. Mostraremos como encontrar todas as EDOs de primeira ordem

$$
y^{\prime}=\omega(x, y)
$$

que admitem um grupo de Lie a um parâmetro cujo gerador infinitesimal é

$$
\mathbf{X}=\xi \partial_{x}+\eta \partial_{y}
$$

A relação entre uma EDO de primeira ordem e seus invariantes diferenciais decorre do próximo teorema.

Teorema 5.1. A EDO (5.49) admite o grupo de simetrias cujo gerador infinitesimal é (5.50) se, e somente se,

$$
\mathbf{X}^{(1)}\left(y^{\prime}-\omega(x, y)\right)=0
$$

sempre que $y^{\prime}=\omega(x, y)$.

O prolongamento de ordem 1 do gerador infinitesimal $\mathbf{X}$ é dado por

$$
\mathbf{X}^{(1)}=\xi(x, y) \partial_{x}+\eta(x, y) \partial_{y}+\eta^{(1)}\left(x, y, y^{\prime}\right) \partial_{y^{\prime}},
$$

com

$$
\eta^{(1)}=\eta_{x}+\left[\eta_{y}-\xi_{x}\right] y^{\prime}-\xi_{y}\left(y^{\prime}\right)^{2}
$$

Note que

$$
\mathbf{X}^{(1)}\left(y^{\prime}-\omega(x, y)\right)=\eta^{(1)}-\mathbf{X} \omega(x, y) .
$$

Logo, segue do Teorema 5.1 e de (5.51) que a EDO (5.49) admite o grupo de simetrias (5.50) se, e somente se, $\eta^{(1)}=\mathbf{X} \omega(x, y)$. Isto é, se, e somente se, $\omega(x, y)$ satisfaz

$$
\eta_{x}+\left(\eta_{y}-\xi_{x}\right) \omega-\xi_{y} \omega^{2}-\xi \omega_{x}-\eta \omega_{y}=0
$$

Observe que (5.52) é a condição de simetria linearizada (4.38). Quando apresentamos (4.38), queríamos para uma dada EDO, determinar um grupo de simetrias. Agora a mesma condição reapareceu ao tentarmos determinar uma EDO que admite um grupo de simetrias conhecido.

As equações características correspondentes a (5.52) são

$$
\frac{d x}{\xi(x, y)}=\frac{d y}{\eta(x, y)}=\frac{d \omega}{\eta_{x}+\left(\eta_{y}-\xi_{x}\right) \omega-\xi_{y} \omega^{2}}
$$

Para caracterizar as soluções de (5.53) em termos de invariantes diferenciais, precisaremos do conceito de integral primeira. 
Definition 5.3. Uma função $\varphi$ não constante e de classe $C^{1}$ é uma integral primeira de (5.53) se ela é constante ao longo de soluções de (5.53). Isto é,

$$
\varphi(x, y, \omega)=k
$$

sempre que $\omega(x, y)$ é solução de (5.53). A constante $k$ depende da solução.

Observe que se $\varphi(x, y, \omega)$ é uma primeira integral de $(5.53)$ e $y^{\prime}=\omega(x, y)$, então $\varphi(x, y, \omega)$ é um invariante de ordem 1 de $\mathbf{X}$. Note que se $u(x, y)$ é um invariante de ordem zero do gerador $\mathbf{X}$, então a função implicitamente determinada por

$$
I_{0}(x, y)=c_{1}
$$

satisfaz

$$
\frac{d y}{d x}=\frac{\eta(x, y)}{\xi(x, y)} \quad \text { ou } \quad \frac{d x}{\xi(x, y)}=\frac{d y}{\eta(x, y)} .
$$

Isto é, qualquer invariante de ordem zero do gerador $\mathbf{X}$ é solução da primeira equação em (5.53). Segue de (5.53) que

$$
\frac{d \omega}{d x}=\frac{\eta_{x}+\left(\eta_{y}-\xi_{x}\right) \omega-\xi_{y} \omega^{2}}{\xi}=\frac{\eta_{x}}{\xi}+\frac{\eta_{y}-\xi_{x}}{\xi} \omega-\frac{\xi_{y}}{\xi} \omega^{2} .
$$

Podemos usar (5.54) para eliminar $y$ e escrever

$$
\frac{\eta(x, y)}{\xi(x, y)}=f_{p}\left(x ; c_{1}\right)
$$

e também para eliminar $y$ em (5.55) e obter

$$
\frac{d \omega}{d x}=A+B \omega+C \omega^{2}
$$

com

$$
\begin{gathered}
A=A\left(x ; c_{1}\right)=\frac{\eta_{x}}{\xi}, \\
B=B\left(x ; c_{1}\right)=\frac{\eta_{y}-\xi_{x}}{\xi}, \\
C=C\left(x ; c_{1}\right)=-\frac{\xi_{y}}{\xi} .
\end{gathered}
$$

Mostraremos agora que $\omega(x, y)=f_{p}\left(x ; c_{1}\right)$ é uma solução particular da EDO de Riccati (5.57). De fato,

$$
\begin{aligned}
\frac{d \omega}{d x} & =\frac{d f_{p}}{d x}=\frac{\left(\eta_{x}+\eta_{y} f_{p}\right) \xi-\eta\left(\xi_{x}+\xi_{y} f_{p}\right)}{\xi^{2}} \\
& =\frac{\eta_{x}}{\xi}+\frac{\eta_{y} f_{p}}{\xi}-\frac{\eta\left(\xi_{x}+\xi_{y} f_{p}\right)}{\xi^{2}}=\frac{\eta_{x}}{\xi}+\frac{\eta_{y} f_{p}}{\xi}-\frac{f_{p}\left(\xi_{x}+\xi_{y} f_{p}\right)}{\xi} \\
& =A+B \omega+C \omega^{2} .
\end{aligned}
$$

A equação (5.57) é uma equação de Riccati e conhecemos uma solução particular dela. Consequentemente, podemos determinar sua solução geral.

$$
\omega=\Xi\left(x ; c_{1}, v\right)
$$


onde $v$ é a constante de integração e $\Xi$ é uma função conhecida. Se resolvemos (5.61) para $v$ e lembramos que $c_{1}=I_{0}(x, y)$, obtemos

$$
v=\Phi(x, y, \omega)
$$

Observe que $\Phi$ é uma primeira integral de (5.53). Se consideramos os casos em que $y^{\prime}=\omega(x, y)$, vemos que $\Phi\left(x, y, y^{\prime}\right)$ é um invariante de ordem 1 de $\mathbf{X}$. Quando nos restringimos ao caso em que $y^{\prime}=\omega(x, y)$, podemos expressar, a partir de (5.61), $v$ em função de $I_{0}(x, y)$. Isto é, $v=\varphi\left(I_{0}(x, y)\right)$.

Teorema 5.2. Se $\psi$ é uma função de classe $C^{1}$ e $\Phi(x, y, \omega)$ é uma primeira integral de (5.53), então $\psi(\Phi(x, y, \omega))$ também é uma primeira integral de (5.53).

Pelo Teorema 5.2, a expressão mais geral para uma solução $\omega$ de (5.52) pode ser obtida implicitamente como

$$
v=\psi(u(x, y))=\psi\left(c_{1}\right)
$$

onde $\psi$ é uma função arbitrária. Consequentemente usando (5.62) e o Teorema 5.2, as EDOs (5.49) que admitem o grupo de simetrias (5.50) são dadas por

$$
\Lambda\left(I_{0}, I_{1}\right)=0
$$

onde $\Lambda$ é uma função arbitrária, $I_{0}$ e $I_{1}$ são invariantes de ordem 0 e 1 do gerador infinitesimal (5.50), respectivamente.

\section{Técnicas de resolução conhecidas vistas sob a ótica de sime- trias}

Nesta seção serão apresentados os métodos usuais para resolver equações diferenciais, vistos a partir da teoria de simetrias de Lie.

\subsection{Fator integrante}

A solução de uma equação diferencial linear não-homogênea de primeira ordem do tipo

$$
\frac{d y}{d x}+F(x) y=G(x)
$$

é

$$
y=e^{-\int_{0}^{x} F d \tau} \int e^{\int_{0}^{x} F d \tau} G(x) d x
$$

Esta solução é obtida multiplicando (6.64) pelo fator integrante $\mu=e^{\int_{0}^{x} F d \tau}$ e integrando ambos os lados da igualdade. O fator integrante $\mu=e^{\int_{0}^{x} F d \tau}$ surge naturalmente se resolvemos (6.64) explorando uma simetria bastante natural no contexto de problemas lineares. Considere a solução $y_{h}=e^{-\int_{0}^{x} F d \tau}$ da equação homogênea

$$
\frac{d y}{d x}+F(x) y=0
$$


A linearidade da equação induz uma simetria para (6.64): o conjunto de transformações $\Gamma_{\varepsilon}:(x, y) \mapsto$ $(\hat{x}, \hat{y})=\left(x, y+\varepsilon y_{h}(x)\right)$. Ou seja, se $y(x)$ é solução da EDO (6.64), então também o é sua imagem sob $\Gamma_{\varepsilon}, y(x)+\varepsilon y_{h}(x)$, pois

$$
\begin{gathered}
\frac{d}{d x}\left[y(x)+\varepsilon y_{h}(x)\right]+F(x)\left[y(x)+\varepsilon y_{h}(x)\right]=y^{\prime}(x)+\varepsilon y_{h}^{\prime}(x)+F(x) y(x)+F(x) \varepsilon y_{h}(x) \\
y(x)+F(x) y(x)+\left[\varepsilon y_{h}(x)^{\prime}+\varepsilon F(x) y_{h}(x)\right]=y(x)+F(x) y(x)=G(x) .
\end{gathered}
$$

Encontrada uma simetria para (6.64), podemos determinar um novo sistema de coordenadas (as coordenadas canônicas) no qual a EDO (6.64) é separável. Inicialmente, determinamos o vetor tangente à órbita do grupo de simetrias:

$$
\xi=\left.\frac{d \hat{x}}{d \varepsilon}\right|_{\varepsilon=0}=0, \quad \eta=\left.\frac{d \hat{y}}{d \varepsilon}\right|_{\varepsilon=0}=\left.\frac{d}{d \varepsilon}\left(y+\varepsilon y_{h}\right)\right|_{\varepsilon=0}=y_{h}
$$

e o seu gerador infinitesimal (4.40):

$$
\mathbf{X}=\xi \frac{\partial}{\partial x}+\eta \frac{\partial}{\partial y}=y_{h} \frac{\partial}{\partial y}
$$

Finalmente, para transformar para coordenadas canônicas $(r, s)$, usamos (4.41):

$$
y_{h} r_{y}=\mathbf{X} r=0, \quad y_{h} s_{y}=\mathbf{X} s=1 \text {. }
$$

Visto que as órbitas de $\Gamma_{\varepsilon}$ são linhas verticais (pois $\xi=0$ ), temos $r=x$. Como $s_{y}=y_{h}(x)^{-1}$, temos $s(x, y)=y_{h}(x)^{-1} y$. Note que em (6.64), temos $\omega(x, y)=-F(x) y+G(x)$. No sistema de coordenadas $(r, s)$, a equação $(6.64)$ se torna

$$
\begin{aligned}
\frac{d s}{d r} & =\frac{s_{x}+\omega(x, y) s_{y}}{r_{x}+\omega(x, y) r_{y}}=-\frac{y_{h}^{\prime} y}{y_{h}^{2}}+\frac{-F(x) y+G(x)}{y_{h}}=-\frac{y\left(-F(x) y_{h}\right)}{y_{h}^{2}}+\frac{-F(x) y+G(x)}{y_{h}} \\
& =\frac{G(x)}{y_{h}(x)}=\frac{G(r)}{y_{h}(r)} .
\end{aligned}
$$

Sua solução é $s(r)=\int \frac{G(r)}{y_{h}(r)} d r=\int \frac{G(r)}{e^{-\int_{0}^{x} F d \tau}} d r$. Voltando às variáveis originais, obtemos

$$
y=e^{-\int_{0}^{x} F d \tau} \int e^{\int_{0}^{x} F d \tau} G(x) d x .
$$

\subsection{Equação homogênea}

Uma EDO homogênea é da forma

$$
\frac{d y}{d x}=F(x, y)
$$

onde $F$ é uma função homogênea de grau zero ${ }^{3}$. A homogeneidade de $F$ e a linearidade do operador derivada sugerem uma simetria para (6.65): o grupo de transformações

$$
(\hat{x}, \hat{y})=\left(e^{\varepsilon} x, e^{\varepsilon} y\right)
$$

\footnotetext{
${ }^{3}$ Dizemos que uma função $f(x, y)$ é homogênea de grau $k$ se: $f(t x, t y)=t^{k} f(x, y)$, para $(x, y)$ pertencente ao domínio de $f$ e $t>0$.
} 
Seu gerador infinitesimal é

$$
\mathbf{X}=\xi(x, y) \partial_{x}+\eta(x, y) \partial_{y}=x \partial_{x}+y \partial_{y},
$$

onde

$$
\begin{aligned}
& \xi=\left.\frac{d \hat{x}}{d \varepsilon}\right|_{\varepsilon=0}=x \\
& \eta=\left.\frac{d \hat{y}}{d \varepsilon}\right|_{\varepsilon=0}=y .
\end{aligned}
$$

Para transformar para coordenadas canônicas $(r, s)$, usamos (4.41):

$$
x r_{x}+y r_{y}=\mathbf{X} r=0, \quad x s_{x}+y s_{y}=\mathbf{X} s=1 .
$$

Usando o método das características, deduzimos que $r=\frac{y}{x}$. Admitindo que $s_{y}=0$,

$$
r=\frac{y}{x} \quad s=\ln |x|
$$

são coordenadas canônicas para (6.65).

Nestas novas coordenadas $r$ e $s,(6.65)$ transforma-se em uma equação separável

$$
\frac{d s}{d r}=\frac{\frac{1}{x}}{-\frac{y}{x^{2}}+F(x, y) \frac{1}{x}}=\frac{\frac{1}{x}}{-\frac{r}{x}+F\left(x \cdot 1, x \cdot \frac{y}{x}\right) \frac{1}{x}}=\frac{1}{F(1, r)-r},
$$

cuja solução é

$$
s=\int \frac{d r}{F(r)-r}+C .
$$

Voltando às variáveis originais, obtemos uma solução de (6.65).

\subsection{Redução de ordem}

Não apresentamos a teoria de Grupos de Lie para EDOs de ordem mais alta, mas vamos ilustrar como o método de simetrias é utilizado para reduzir a ordem de uma EDO de ordem 2.

Exemplo 6.1. A equação diferencial homogênea de segunda ordem

$$
y^{\prime \prime}+p(x) y^{\prime}+q(x) y=0
$$

é invariante sob o conjunto de transformações $\Gamma_{\varepsilon}(x, y) \mapsto(\hat{x}, \hat{y})=\left(x, e^{\varepsilon} y\right)$.

De fato, se o fator $e^{\varepsilon}$ é colocado em evidência, obtemos

$$
\left(e^{\varepsilon} y\right)^{\prime \prime}+p(x)\left(e^{\varepsilon} y\right)^{\prime}+q(x)\left(e^{\varepsilon} y\right)=e^{\varepsilon}\left(y^{\prime \prime}+p(x) y^{\prime}+q(x) y\right)=0 .
$$

O gerador infinitesimal do grupo de simetrias $\Gamma_{\varepsilon}$ é

$$
\mathbf{X}=\xi(x, y) \partial_{x}+\eta(x, y) \partial_{y}=y \partial_{y},
$$


onde

$$
\begin{gathered}
\xi=\left.\frac{d \hat{x}}{d \varepsilon}\right|_{\varepsilon=0}=0 \\
\eta=\left.\frac{d \hat{y}}{d \varepsilon}\right|_{\varepsilon=0}=y .
\end{gathered}
$$

Vamos determinar as coordenadas canônicas. Como $\xi=0$, as órbitas de $\Gamma_{\varepsilon}$ são linhas verticais e tomamos $r=x$. Para determinar $s$, devemos resolver

$$
y s_{y}=\mathbf{X} s=1 .
$$

Isto é, $s=\ln |y| \Rightarrow y=e^{s}$. Como $r=x$, faremos uma mudança de coordenada apenas na variável dependente. Temos

$$
\begin{gathered}
s^{\prime}=\frac{d s}{d x}=\frac{d}{d x}(\ln |y|)=\frac{1}{y} \cdot y^{\prime} \\
s^{\prime \prime}=-\frac{1}{y^{2}} \cdot\left(y^{\prime}\right)^{2}+\frac{1}{y} \cdot y^{\prime \prime} . \\
p(x) s^{\prime}=\frac{1}{y} \cdot p(x) y^{\prime} \\
s^{\prime \prime}+\left(s^{\prime}\right)^{2}=\frac{1}{y} \cdot y^{\prime \prime} .
\end{gathered}
$$

Na nova coordenada temos, então

$$
s^{\prime \prime}+s^{\prime 2}+p(x) s^{\prime}+q(x)=0 .
$$

Como (6.67) não depende de $s$, a mudança de variáveis $z=s^{\prime}$ reduz a ordem da EDO (6.67):

$$
z^{\prime}+z^{2}+p(x) z+q(x)=0 .
$$

a uma EDO de primeira ordem.

\section{Considerações finais}

As simetrias de Lie permitem unificar métodos de resolução de EDOs apresentados em cursos de graduação. Seu conhecimento permite também, se formos capazes de definir uma simetria adequada, a resolução de algumas EDOs que não se enquadram nas famílias para as quais conhecemos um procedimento de resolução. Os métodos são apresentados de maneira natural e deixam de parecer retirados de uma sacola de truques.

\section{Agradecimentos}

Os autores agradecem o apoio da FAPERJ, CAPES e CNPq a este trabalho. 


\section{Referências}

[1] Cheb-Terrab, E. S. and Kolokolnikov, T. First-order ordinary differential equations, symmetries and linear transformations, European Journal of Applied Mathematics, 14(2), 2003, p. 231-246.

[2] Hydon, Peter E. Symmetry Methods for Differential Equations: A Beginner's Guide, Cambridge University Press, 2000.

[3] Lie, Sophus. Theorie der transformationsgruppen I, Mathematische Annalen, 16(4), p. 441-528, Springer, 1880 .

[4] Starrett, John. Solving Differential Equations by Symmetry Groups, The American Mathematical Monthly, 114(9), 2007, p. 778-792.

[5] Yap, Shirley Llamado. Differential Equations-Not Just a Bag of Tricks!, Mathematics Magazine, 83(1), 2010, p. 3-14.

[6] Steinhour, Ruth A. The Truth About Lie Symmetries: Solving Differential Equations With Symmetry Methods, 2013. 